\title{
TAKAGI-SUGENO TYPE FUZZY INFERENCE MODULE IN THE CASE OF INDEPENDENT LINGUISTIC VARIABLES
}

\section{D.T.MUHAMEDIYEVA \& Y.SH.BAKHRAMOVA}

Tashkent University of Information Technologies Named After Muhammad al-Khwarizmi, Amir Temur Street, Tashkent,

Uzbekistan

\begin{abstract}
The simultaneous identification of fuzzy rules and the adaptation of membership functions turned out to be very difficult or even impossible. This paper considers fuzzy neural networks that can solve this problem. We will discuss two types of fuzzy control modules that are based on the rules proposed by Takagi and Sugeno. These modules differ in the way they formulate conclusions of fuzzy rules. The result of the proposed approach will be expressed, firstly, in the implementation of the procedure for inference by the neural network of the corresponding structure and, secondly, in the display of the parameters of this procedure by the weights of connections.

KEYWORDS: Fuzzy Set Theory, Neural Networks, Membership Function, Activation Function, Neural Network Calculations.
\end{abstract}

Received: Jun 08, 2020; Accepted: Jun 28, 2020; Published: Sep 11, 2020; Paper Id.: IJMPERDJUN20201163

\section{INTRODUCTION}

Due to the limited possibilities of traditional methods of mathematical modeling, when solving poorly formalized problems, including those associated with risk, data mining technologies are used. They are based on the methods of artificial intelligence and, especially, methods of soft computing (Soft Calculation, Soft Computing) and the direction of Computational Intelligence arising on this theoretical and methodological basis - intelligent computing technologies. The main components of these areas are the theory of fuzzy sets, fuzzy inference, genetic algorithms, artificial neural networks and neural network computing. Computational Intelligence technology allows you to get solutions with an accuracy acceptable for practice, by training on the available source data, available in a limited, incomplete volume, and also presented in a qualitative form [1-3].

The represented fuzzy neural network will be able to simultaneously form fuzzy rules and adapt membership functions by modifying the weights of the connections in the learning process and - most importantly for this, the classic back propagation algorithm will be used [1,4].

\section{NEURAL IMPLEMENTATION OF THE MEMBERSHIP FUNCTION}

In the structures, elements were used that implement the input accessory functions in the form of Gaussian curves or triangular, but the ways of their implementation were not analyzed. It was also noted that these elements can be replaced with simple neural networks of a certain design, which, after the necessary training, will display rather complex membership functions [1,5-7].

Let's consider a very simple implementation of a single membership $\pi$-class function in the form of a two-layer neural network. The result of its functioning is the degree of belonging of the $i$-th input $\bar{x}_{i}$-signal to the 
$k$-th fuzzy set $A_{i}^{k}$.

Layer L1. In the first layer, you can distinguish two neurons with a sigmoid activation function, which has the form

$$
f(x)=\frac{1}{1+\exp (-x)}
$$

Offsets in the form of weights $\bar{x}_{a}^{(k)}$ and $\bar{x}_{b}^{(k)}$ are also applied to the adder inputs along with the input signal. The following weights $h_{a}^{(k)}$ and $h_{b}^{(k)}$ provide scaling of the signals. Since the functioning of the nodes designated «sgm» is described by the expression (1), we get the output of this layer

$$
\begin{aligned}
& f_{a}^{(k)}(x)=\frac{1}{1+\exp \left[-h_{a}^{(k)}\left(x+\bar{x}_{a}^{(k)}\right)\right]} \\
& f_{b}^{(k)}(x)=\frac{1}{1+\exp \left[-h_{b}^{(k)}\left(x+\bar{x}_{b}^{(k)}\right)\right]} .
\end{aligned}
$$

It is clear that the weights of the links $\bar{x}_{a}^{(k)}$ and $\bar{x}_{b}^{(k)}$, as well as $h_{a}^{(k)}$ and $h_{b}^{(k)}$ are parameters that modify, respectively, the placement of centers and the curvature of two sigmoid functions relative to the input signal $\mathrm{x}$.

Layer L2. This layer contains a single neuron with a linear activation function. It combines the sigmoid functions obtained in the previous layer and creates a membership function for the fuzzy set based on them $A^{K}$ :

$$
\mu_{A^{k}}(x)=f_{a}^{(k)}(x)-f_{b}^{(k)}(x)
$$

Sigmoid functions have opposite signs and are shifted relative to each other. Thus, the resulting function (4) is characterized by two weight vectors:

$$
\bar{x}^{(k)}=\left[x_{a}^{(k)}, x_{b}^{(k)}\right]^{T} \text { и } h^{(k)}=\left[h_{a}^{(k)}, h_{b}^{(k)}\right]^{T} .
$$

\section{TAKAGI-SUGENO MODULES}

In many cases, the classic base of fuzzy rules of the form was used

$$
\left.R^{(k)}: I F\left(x_{1} \text { this } A_{1}^{k} A N D \text {...AND } x_{n} \text { this } A_{n}^{k}\right) \text { THEN (have it } B^{k}\right) .
$$

Consider a slightly different approach proposed by Takagi and Sugeno. It consists in the fact that the conclusions of the rules are not represented as belonging to the output variable to fuzzy sets, but in the form of functional dependencies

$$
\begin{gathered}
R^{(k)}: \text { IF }\left(x_{1} \text { this } A_{1}^{k} \text { AND ...AND } x_{n} \text { this } A_{n}^{k}\right) \text { THEN } \\
y=f^{(k)}\left(x_{1}, \ldots, x_{n}\right) .
\end{gathered}
$$


In this case, during the output process, each rule will generate its own numerical value of the control action.

The proposed fuzzy control module is divided into two parts, corresponding to the conditions and conclusions. How to implement conclusions are constructed differently: they are based on dependencies defined by rules of type (6). The following sections describe both the General method for constructing rule conditions and two examples of implementing conclusions.

\section{IMPLEMENTATION CONDITIONS}

The part of the structure that corresponds to the conditions is unchanged for all types of fuzzy control modules.

Layer L1. The task of this layer is to calculate the degree of belonging of the input data to the corresponding fuzzy sets. Elements are represented by single symbols, but simple networks can be hidden under such designations.

The vectors of parameters $\bar{x}_{i}^{(k)}$ and $h_{i}^{(k)}$ and specify the location and shape of the membership functions. They should be chosen so that the functions depending on them uniformly cover the input space.

Layer L2. This layer determines the degree to which the values of the input signals correspond to the conditions of the rules. The relationship between their inputs and outputs is

$$
\begin{aligned}
& \tau_{k}=\prod_{i=1, \ldots, n} \mu_{A_{i}^{k}}\left(\bar{x}_{i}\right), \\
& \tau_{k}=\prod_{i=1, \ldots, n} \mu_{A_{i}^{k}}\left(\bar{x}_{i}\right)=\frac{\tau_{k}}{\sum_{i=1}^{N} \tau_{i}},
\end{aligned}
$$

Where $\mu_{A_{i}^{k}}\left(\bar{x}_{i}\right)$ - is the degree of compliance of the input data with the conditions of the rules, $\tau_{k}$-is the degree of activity of the $k$-th rule, $\bar{\tau}_{k}$-is the normalized value $\tau_{k}$. Therefore, it can be argued that the outputs of this layer represent normalized degrees of rule activity.

Connections with the previous layer are established so as to obtain all possible combinations of membership functions of both input signals. Let us assume that the input space $N_{1}$ is evenly divided by the membership functions for the signal $x_{1}$ and, accordingly, $N_{2}$ by the membership functions for the signal $x_{2}$. In other words, we define $N_{1}$ fuzzy sets for the first input signal: $A_{1}^{1}, \ldots, A_{1}^{N_{1}}$ and $A_{2}^{1}, \ldots, A_{2}^{N_{2}}$ - for the second signal. As a result, we obtain conditions $N_{1} \times N_{2}$ for the rules of the form

$$
R^{\left(n_{1}, n_{2}\right)}: \operatorname{IF}\left(x_{1} \text { is } A_{1}^{n_{1}} \text { AND } x_{2} \text { is } A_{1}^{n_{2}}\right) \text { THEN ..., }
$$

Where $n_{1}=1, \ldots, N_{1}$ and $n_{2}=1, \ldots, N_{2}$.

The degree of compliance with the condition of each rule is calculated as the normalized product of the values of the input variables belonging to these fuzzy sets. For example, the output of the element denoted $\hat{\tau}_{k}$ is the normalized 
product of the degrees of membership in fuzzy sets $A_{1}^{1}$ and $A_{2}^{1}$. Therefore, this node passes the value of the degree of compliance of the input data with the fuzzy rule condition $R_{1,1}$.

\section{THE IMPLEMENTATION OF THE CONCLUSIONS}

Consider two types of fuzzy control modules that implement different fuzzy output methods. The differences are in the way conclusions are formulated; they can be expressed as a constant (type I) or a first-order linear equation (type II),

Type I (Conclusion-constant). The component corresponding to the conclusion is expressed as a constant. Fuzzy inference is represented by a base of fuzzy rules of the form

$$
R^{(k)}: \operatorname{IF}\left(x_{1} \text { this } A_{1}^{k} \text { AND...AND } x_{n} \text { this } A_{n}^{k}\right) \text { THEN } y=c^{(k)}
$$

For $k=1, \ldots, N$, where $R(k)$ denotes the $k$-th fuzzy rule. $A_{1}^{k}$ and $A_{n}^{k}$-these are fuzzy sets of condition components, $c^{(k)}$ - a constant, and $N$ - is the number of rules. To get $\bar{y}$ a quantitative value of the control effect, you can use the method of defuzzification by the middle center

$$
\bar{y}=\sum_{k=1}^{N} \hat{\tau}_{k} c(k)
$$

Where $\hat{\tau}_{k}$ is defined by the formula (8).

The first and second layers are «responsible» for the conditions. Rule conclusions and the defuzzification method are implemented in the third layer, which will be briefly described later.

Layer L3. This layer implements expression (11). The signal at its output is the sum of the products of the weights $w_{c}^{(k)}$ and the normalized degrees of activity of the rules $\hat{\tau}_{k}$. The link weights indicated by the symbol $w_{c}^{(k)}$ correspond to a constant $c^{(k)}$ in the rules of the form (10). They must have zero initial values, which reflects the fact that there are no conclusions before the network training begins. Therefore, it can be argued that modifying these weights in the learning process is equivalent to building rules.

Type II (Conclusion-linear equation). Conclusions in the second type of fuzzy control module are expressed by a linear equation. In this case, the database of fuzzy rules is presented in the form

$$
\begin{gathered}
R^{(k)}: I F\left(x_{1} \text { this } A_{1}^{k} \text { AND ...AND } x_{n} \text { this } A_{n}^{k}\right) \text { THEN } \\
y=f^{(k)}\left(x_{1}, \ldots, x_{n}\right)
\end{gathered}
$$

Where

$$
f^{(k)}\left(x_{1}, \ldots, x_{n}\right)=c_{0}^{(k)}+c_{1}^{(k)} x_{1}+\ldots+c_{n}^{(k)} x_{n}
$$

For $k=1, \ldots, N, c_{i}^{(k)}(i=0, \ldots, n)$ are constants (the other notations match those used for type I inference). 
If the same method is used for defuzzification as in the previous case, the expression defining the quantitative value of the control action takes the form

$$
\bar{y}=\frac{\sum_{k=1}^{N} \tau_{k} f^{(k)}\left(x_{1}, \ldots, x_{n}\right)}{\sum_{k=1}^{N} \tau_{k}}=\sum_{k=1}^{N} \hat{\tau}_{k} f^{(k)}\left(x_{1}, \ldots, x_{n}\right)
$$

In the module, you can select layers that implement rule conditions (L1 and L2), conclusions (L3 and L4), and a defuzzification block (layers L5 and L6). Let's take a closer look at how to implement a linear function.

Layer L3. The link weights $w_{s}=\left[w_{s}^{1}, w_{s}^{2}\right]^{T}$ of this layer scale the input signals. They take values that are the reverse of the maximum absolute values of the corresponding input signals:

$$
w_{s}^{i}=\frac{1}{\max \left(\left|x_{i}\right|\right)}
$$

Due to this approach, normalized signals located in the range $[-1,1]$ are formed at the outputs of nodes of this layer. These weights are not modified during training.

Layer L4. In this layer, the resulting value is output according to each rule. In particular, according to expression (13), a linear dependence of the form is applied in the two-dimensional case

$$
f^{(k)}\left(x_{1}, x_{2}\right)=c_{0}^{(k)}+c_{1}^{(k)} x_{1}+c_{2}^{(k)} x_{2}
$$

Using the vector of normalizing coefficients of the previous layer $\mathrm{w}_{\mathrm{E}}$ leads to the fact that the values of link weights $w_{c_{1}}^{(k)}, w_{c_{2}}^{(k)}, w_{c_{3}}^{(k)}$ become independent of the range of values of input variables.

The products of the weights $w_{s}^{1}$ and $w_{c_{1}^{(k)}}$, as well as $w_{s}^{2}$ and $w_{c_{2}^{(k)}}$ form the coefficients $c_{1}^{(k)}$ and $c_{2}^{(k)}$. The weight $w_{c_{n}^{(k)}}$ directly corresponds to the coefficient $c_{0}^{(k)}$. During the training process, only weights $w_{c_{0}^{(k)}}, w_{c_{1}^{(k)}}, w_{c_{2}^{(k)}}$ are modified to identify fuzzy rules (the vector $w_{s}$ does not change). The initial values of the weights $w_{c_{0}^{(k)}}, w_{c_{1}^{(k)}}, w_{c_{2}^{(k)}}$ must be zero.

The defuzzification block is implemented in layers L5 and L6.

Layers L5 and L6. In layer L5, the product of the normalized degree of compliance with the conditions $\bar{\tau}_{k}$ and the function $f^{(k)}\left(x_{1}, x_{2}\right)$, contained in the conclusion component is calculated. Thus, in this layer, the condition of each rule is combined with its corresponding conclusion. As a result, quantitative values of control actions are formed at the outputs of the L5 layer elements, which are output for each fuzzy rule.

In layer L6, the results obtained in the previous layer are aggregated and the final value of the control action is generated. 


\section{COMPUTATIONAL EXPERIMENT}

A method for assessing forecasting based on approximating models of fuzzy inference with fuzzy given initial information about risk assessment is proposed.

The construction of fuzzy risk models usually begins with a qualitative analysis aimed at identifying risks. The achievement of this goal is divided into the solution of the following tasks: identification of the entire spectrum of risks; description of risks; classification and grouping of risks; analysis of the initial assumptions.

The second and most difficult stage of building fuzzy risk models is quantitative risk analysis, the purpose of which is to measure risk, which leads to the solution of the following tasks: formalization of uncertainty; calculation of risks; risk assessment; accounting for risks.

At the third stage, fuzzy risk models are built and the start of its implementation.

The fourth stage is control.

The risk assessment model is described using fuzzy inference rules:

$$
\bigcup_{p=1}^{k_{j}}\left(\bigcap_{i=1}^{n} x_{i}=a_{i, j p}-c \text { весом } w_{j p}\right) \rightarrow r=f\left(x_{1}, x_{2}, \ldots, x_{n}\right) \text {. }
$$

Here $a_{i, j p}$ is the linguistic term used to evaluate the variable $x_{i}$ in the line with the number $j p$;

$w_{j p}$ - weighting coefficient of the rule with the serial number $j p$;

$r=f\left(x_{1}, x_{2}, \ldots, x_{n}\right)-$ output of a fuzzy rule.

Fuzzy logical equations (such as if <input>, then <output>) together with membership functions of fuzzy terms, make it possible to make a decision on risk assessment using the following algorithm:

1. The values of the object state parameters are fixed:

$$
X^{*}=\left(x_{1}^{*}, x_{2}^{*}, \ldots, x_{n}^{*}\right) \text {. }
$$

2. The values of the membership functions $\mu^{j}\left(x_{i}^{*}\right)$ are determined for fixed values of the parameters $x_{i}^{*}, i=\overline{1, n}$.

3. Using logical equations, the values of the membership functions $\mu^{r_{j}}\left(x_{1}^{*}, x_{2}^{*}, \ldots, x_{n}^{*}\right)$ at the state vector $X^{*}=\left(x_{1}^{*}, x_{2}^{*}, \ldots, x_{n}^{*}\right)$ are calculated.

4. A solution $r_{j}^{*}$ is determined for which:

$\mu^{r_{j}^{*}}\left(x_{1}^{*}, x_{2}^{*}, \ldots, x_{n}^{*}\right)=\max _{j=1, n}\left[\mu^{r_{j}}\left(x_{1}^{*}, x_{2}^{*}, \ldots, x_{n}^{*}\right)\right]$. 
The essence of training consists in the selection of such parameters of the membership functions that minimize the difference between the results of the neuro-fuzzy approximation and the real behavior of the object. A system of recurrent relations is used for training:

$$
\begin{aligned}
& b_{k}^{j}(t+1)=b_{k}^{j}(t)-\eta\left(r_{t}-\hat{r}_{t}\right) \frac{r_{j} \sum_{i=1}^{m} \mu^{r_{i}}\left(r_{i}\right)-\sum_{i=1}^{m} r_{i} \mu^{r_{i}}\left(r_{i}\right)}{\left(\sum_{i=1}^{m} \mu^{r_{i}}\left(r_{i}\right)\right)^{2}} \frac{1}{\mu^{k}\left(x_{i}^{j}\right)} \prod_{i=1}^{n} \mu^{j}\left(x_{i}^{j}\right) \frac{2 c_{k}^{j}\left(x_{i}^{j}-b_{k}^{j}\right)^{2}}{\left(\left(c_{k}^{j}\right)^{2}+\left(x_{i}^{j}-b_{k}^{j}\right)^{2}\right)^{2}}, \\
& c_{k}^{j}(t+1)=c_{k}^{j}(t)-\eta\left(r_{t}-\hat{r}_{t}\right) \frac{r_{j} \sum_{i=1}^{m} \mu^{r_{i}}\left(r_{i}\right)-\sum_{i=1}^{m} r_{i} \mu^{r_{i}}\left(r_{i}\right)}{\left(\sum_{i=1}^{m} \mu^{r_{j}}\left(r_{i}\right)\right)^{2}} \frac{1}{\mu^{k}\left(x_{i}^{j}\right)} \prod_{i=1}^{n} \mu^{j}\left(x_{i}^{j}\right) \frac{2\left(c_{k}^{j}\right)^{2}\left(x_{i}^{j}-b_{k}^{j}\right)}{\left(\left(c_{k}^{j}\right)^{2}+\left(x_{i}^{j}-b_{k}^{j}\right)^{2}\right)^{2}} .
\end{aligned}
$$

The training algorithm for the neuro-fuzzy network consists of two phases. At the first phase, the model value of the object output $(r)$ is calculated, which corresponds to the given network architecture. In the second phase, the residual value $\left(E_{t}\right)$ is calculated and the parameters of the membership functions are recalculated.

Three types of models for assessing the risk of crop shortfall have been developed, based on fuzzy inference rules.

1. Risk assessment model, the output of which is expressed by a linear relationship.

If $x_{1}^{1}=H$ and $x_{2}^{1}=H$ and $x_{3}^{1}=H$ and $x_{4}^{1}=C$

Then $r_{1}=0,33-0,05 \frac{\sum_{j=1}^{n} \mu\left(x_{1}^{1 j}\right) x_{1}^{1 j}}{\sum_{j=1}^{n} \mu\left(x_{1}^{1 j}\right)}-0,02 \frac{\sum_{j=1}^{n} \mu\left(x_{2}^{1 j}\right) x_{2}^{1 j}}{\sum_{j=1}^{n} \mu\left(x_{2}^{1 j}\right)}-0,21 \frac{\sum_{j=1}^{n} \mu\left(x_{3}^{1 j}\right) x_{3}^{1 j}}{\sum_{j=1}^{n} \mu\left(x_{3}^{1 j}\right)}-0,1 \frac{\sum_{j=1}^{n} \mu\left(x_{4}^{1 j}\right) x_{4}^{1 j}}{\sum_{j=1}^{n} \mu\left(x_{4}^{1 j}\right)}$.

If $x_{1}^{2}=H$ and $x_{2}^{2}=H$ and $x_{3}^{2}=C$ and $x_{4}^{2}=C$

Then $r_{2}=0,257-0,0393 \frac{\sum_{j=1}^{n} \mu\left(x_{1}^{2 j}\right) x_{1}^{2 j}}{\sum_{j=1}^{n} \mu\left(x_{1}^{2 j}\right)}-0,112 \frac{\sum_{j=1}^{n} \mu\left(x_{4}^{2 j}\right) x^{2 j}}{\sum_{j=1}^{n} \mu\left(x_{4}^{2 j}\right)}$

If $x_{1}^{3}=H$ and $x_{2}^{3}=C$ and $x_{3}^{3}=H a n d x_{4}^{3}=C$

Then $r_{3}=0,18-0,01 \frac{\sum_{j=1}^{n} \mu\left(x_{1}^{3 j}\right) x_{1}^{3 j}}{\sum_{j=1}^{n} \mu\left(x_{1}^{3 j}\right)}-0,07 \frac{\sum_{j=1}^{n} \mu\left(x_{2}^{3 j}\right) x_{2}^{3 j}}{\sum_{j=1}^{n} \mu\left(x_{2}^{3 j}\right)}-0,05 \frac{\sum_{j=1}^{n} \mu\left(x_{3}^{3 j}\right) x_{3}^{3 j}}{\sum_{j=1}^{n} \mu\left(x_{3}^{3 j}\right)}-0,111 \frac{\sum_{j=1}^{n} \mu\left(x_{4}^{3 j}\right) x_{4}^{3 j}}{\sum_{j=1}^{n} \mu\left(x_{4}^{3 j}\right)}$.

If $x_{1}^{4}=H$ and $x_{2}^{4}=C$ and $x_{3}^{4}=C$ and $x_{4}^{4}=C$ 
Then $r_{4}=0,26-0,02 \frac{\sum_{j=1}^{n} \mu\left(x_{1}^{4 j}\right) x_{1}^{4 j}}{\sum_{j=1}^{n} \mu\left(x_{1}^{4 j}\right)}-0,05 \frac{\sum_{j=1}^{n} \mu\left(x_{2}^{4 j}\right) x_{2}^{4 j}}{\sum_{j=1}^{n} \mu\left(x_{2}^{4 j}\right)}-0,03 \frac{\sum_{j=1}^{n} \mu\left(x_{3}^{4 j}\right) x_{3}^{4 j}}{\sum_{j=1}^{n} \mu\left(x_{3}^{4 j}\right)}-0,134 \frac{\sum_{j=1}^{n} \mu\left(x_{4}^{4 j}\right) x_{4}^{4 j}}{\sum_{j=1}^{n} \mu\left(x_{4}^{4 j}\right)}$.

If $x_{1}^{5}=H$ and $x_{2}^{5}=C$ and $x_{3}^{5}=$ Band $x_{4}^{5}=C$

Then $r_{5}=0,202-0,10 \frac{\sum_{j=1}^{n} \mu\left(x_{1}^{5 j}\right) x_{1}^{5 j}}{\sum_{j=1}^{n} \mu\left(x_{1}^{5 j}\right)}-0,08 \frac{\sum_{j=1}^{n} \mu\left(x_{2}^{5 j}\right) x_{2}^{5 j}}{\sum_{j=1}^{n} \mu\left(x_{2}^{5 j}\right)}-0,04 \frac{\sum_{j=1}^{n} \mu\left(x_{3}^{5 j}\right) x_{3}^{5 j}}{\sum_{j=1}^{n} \mu\left(x_{3}^{5 j}\right)}-0,12 \frac{\sum_{j=1}^{n} \mu\left(x_{4}^{5 j}\right) x_{4}^{5 j}}{\sum_{j=1}^{n} \mu\left(x_{4}^{5 j}\right)}$.

\section{CONCLUSIONS}

The structures of fuzzy control modules presented in this section are characterized by the following important properties:

- The formation of fuzzy rules and the refinement of the membership functions are carried out in parallel and fully automatically;

- The presented two ways of implementing fuzzy inference should be considered only as the simplest examples.

Experiments with other possible solutions will definitely bear fruit in the form of alternative, but no less interesting structures.

\section{REFERENCES}

1. Zadeh L. A. Fuzzy Sets // Information and Control. 1965. -Vol. 8, №3.-P. 338-353.

2. Muhamediyeva D.T. and Niyozmatova N.A. 2019 Problems of constructing models of intellectual analysis ofstates of weakly formalizable processes. Journal of Physics: 1210.

3. Muhamediyeva D.T. and Niyozmatova N.A. 2019Approaches to solving the problem of fuzzy parametric programming in weakly structured objects. Journal of Physics. 1260.

4. Muhamediyeva D.T. and Sayfiyev J. 2019 Approaches to the construction of nonlinear models in fuzzy environment. Journal of Physics. 1260.

5. Singh, D., Singh, M., \&Hakimjon, Z. (2019). Geophysical application for splines. In SpringerBriefs in Applied Sciences and Technology (pp. 55-63). SpringerVerlag.https://doi.org/10.1007/978-981-13-2239-6_7.

6. Fazilov, S., Mamatov, N., Samijonov, A., Abdullaev, S. (2019).Reducing the dimensionality of feature space in pattern recognition tasks Journal of Physics: Conference Series 1441(1).

7. Sotvoldiev D., Muhamediyeva D.T., Juraev Z. Deep learning neural networks in fuzzy modeling // IOP Conf. Series:Journal of Physics: Conference Series 1441 (2020) 012171.DOI: https://doi.org/10.1088/1742-6596/1441/1/012171.

8. K. Punniakrishnan \& K. Kadambavanam, "On Intuitionistic Fuzzy Inventory Models without Allowing Storage Constraint ", IMPACT: International Journal of Research in Engineering \& Technology (IMPACT: IJRET), Vol. 2, Issue 2, pp. 155-166

9. V. Anusuya \& R. Sathya, "An Algorithmic Approach for Shortest Path Problem by Possibility Measure with Type-2 Fuzzy Number “, International Journal of Applied Mathematics \& Statistical Sciences (IJAMSS), Vol. 4, Issue 1, pp. 1-10 
10. G. Kumaresan \& P. Rajakumar, "Predictive Analytics Using Big Data: A Survey “, BEST: International Journal of Management, Information Technology and Engineering (BEST: IJMITE), Vol. 3, Issue 9, pp. 61-68

11. P. S. Dhabe \& N. R. Yengul , "GPU Parallelization of Fuzzy Min-Max Clustering Neural Network for Pattern Recognition ", IMPACT: International Journal of Research in Engineering \& Technology (IMPACT: IJRET), Vol. 5, Issue 6, pp. 55-68 

